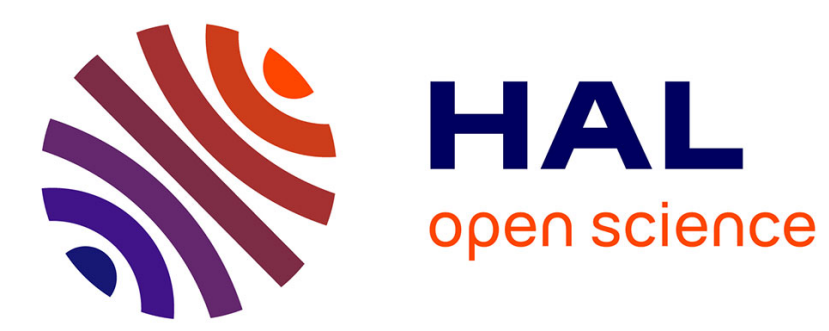

\title{
On the sextet polynomial of fullerenes
}

Jean-Sébastien Sereni, Matej Stehlík

\section{To cite this version:}

Jean-Sébastien Sereni, Matej Stehlík. On the sextet polynomial of fullerenes. Journal of Mathematical Chemistry, 2010, 47 (3), pp.1121-1128. hal-00487101

\section{HAL Id: hal-00487101 https://hal.science/hal-00487101}

Submitted on 27 May 2010

HAL is a multi-disciplinary open access archive for the deposit and dissemination of scientific research documents, whether they are published or not. The documents may come from teaching and research institutions in France or abroad, or from public or private research centers.
L'archive ouverte pluridisciplinaire HAL, est destinée au dépôt et à la diffusion de documents scientifiques de niveau recherche, publiés ou non, émanant des établissements d'enseignement et de recherche français ou étrangers, des laboratoires publics ou privés. 


\title{
On the sextet polynomial of fullerenes
}

\author{
Jean-Sébastien Sereni* Matěj Stehlík ${ }^{\dagger}$
}

\begin{abstract}
We show that the sextet pattern count of every fullerene is strictly smaller than the Kekule structure count. This proves a conjecture of Zhang and He [J. Math. Chem. 38(3):2005, p. 315-324].
\end{abstract}

\section{Introduction}

Fullerenes are cage polyhedral carbon molecules such that all faces are pentagons and hexagons. The icosahedral $\mathrm{C}_{60}$, known as the buckminsterfullerene, was predicted by Osawa [14, and discovered by Kroto et al. [10]. A fullerene with no adjacent pentagons is said to satisfy the isolated pentagon rule (IPR), and is called an IPR fullerene. The buckminsterfullerene is the smallest IPR fullerene, and all the most stable fullerenes are IPR fullerenes. Since the discovery of the first fullerene molecule, fullerenes have been studied by scientists across many disciplines.

Various properties of fullerene molecules can be studied using mathematical tools and results. Thus, fullerene graphs were defined as 3-connected cubic plane graphs with all faces of size five or six; see e.g. the graph $I_{h}$ in Figure 1. Such graphs are suitable models for fullerene molecules: carbon atoms are represented by vertices of the graph, whereas the edges represent bonds between adjacent atoms. It is known that there exists a fullerene graph on $n$ vertices for every even $n \geq 20, n \neq 22$. See the monograph of Fowler and Manolopoulos [3] for more information on fullerenes.

Since carbon atoms in stable molecules are tetravalent, one of the three $\sigma$ bonds of every atom in a fullerene molecule should be augmented by a $\pi$-bond to create a carbon-carbon double bond. A Lewis structure that localizes the

${ }^{*}$ CNRS (LIAFA, Université Denis Diderot), Paris, France and Department of Applied Mathematics (KAM), Faculty of Mathematics and Physics, Charles University, Prague, Czech Republic (sereni@kam.mff.cuni.cz).

${ }^{\dagger}$ LIAFA, Université Denis Diderot (Paris 7), Paris, France (matej@liafa.jussieu.fr). 
$\sigma$ - and $\pi$-bonds to at most two atoms per bond is called a Kekulé structure. It corresponds to the notion of perfect matchings in fullerene graphs: a perfect matching in a graph $G$ is a set $M$ of edges of $G$ such that every vertex of $G$ is incident with precisely one edge of $M$. We let $\operatorname{pm}(G)$ be the number of perfect matchings of $G$; this corresponds to the number of Kekule structures in a fullerene, also known as the Kekulé count $K$. The Kekulé count is an important parameter in theoretical chemistry of benzenoid hydrocarbons, for it is an empirically established fact that the total $\pi$-electron energy of benzenoid hydrocarbons is a linear function of the Kekulé count (see e.g. the book by Gutman and Cyvin [5]).

Let $M$ be a perfect matching in a fullerene graph $G$. A hexagonal face is resonant (with respect to $M$ ) if it is incident with precisely three edges in $M$. A (possibly empty) set of pairwise vertex-disjoint resonant hexagons is a sextet pattern (or resonant pattern), and the maximum size of a sextet pattern in $G$ is the Clar number of $G$. The concept of sextet pattern originates from Clar's aromatic theory [2]. The sextet polynomial $B_{G}(x)$ of a benzenoid system $G$ is defined [7, 13] as

$$
B_{G}(x):=\sum_{k=0}^{m} r(G, k) x^{k},
$$

where $r(G, k)$ is the number of sextet patterns of $G$ of size $k$, and $m$ is the Clar number of $G$. We define $\mathscr{R}(G)$ to be the set of resonant patterns of $G$. Note that $B_{G}(1)=|\mathscr{R}(G)|$.

It has been shown [4, 6, 19] that the inequality

$$
B_{G}(1) \leq \operatorname{pm}(G)
$$

holds for all benzenoid systems, with equality for catacondensed benzenoid systems [7, 13. The inequality (1) was subsequently extended to all planar bipartite graphs [8, 16], and eventually to all planar graphs [20]. However, the Kekulé count is a poor indicator of $\pi$-electronic stability for fullerenes: although experiments show that the buckminsterfullerene is the most stable isomer of $\mathrm{C}_{60}$, it was shown by Austin et al. [1] that there are 20 isomers of $\mathrm{C}_{60}$ with a higher Kekulé count than the buckminsterfullerene.

This led Zhang and He [20] to conjecture that no fullerene graph achieves equality in (1).

Conjecture 1 (Zhang and He). For every fullerene graph $G$,

$$
B_{G}(1)<\operatorname{pm}(G)
$$


Zhang and He [20] verified Conjecture 1 for fullerene graphs containing at least one pair of adjacent pentagons, i.e. for fullerene graphs violating the IPR. The purpose of this paper is to prove the conjecture for all fullerenes, including the most stable ones. Namely, we prove the following result.

Theorem 2. For every fullerene graph $G$,

$$
B_{G}(1)<\operatorname{pm}(G)
$$

\section{Notation}

Given a graph $G$, we let $V(G)$ and $E(G)$ be its vertex set and edge set, respectively. For $H \subseteq V(G) \cup E(G)$, we let $G-H$ be the graph obtained from $G$ by removing the elements in $H$. Let $X \subset V(G)$. We define $G[X]$ to be the subgraph of $G$ induced by the vertices in $X$. We let $\delta(X)$ be the set of edges of $G$ with exactly one end-vertex in $X$.

A $k$-edge-cut of $G$ is a set $Y$ of $k$ edges of $G$ for which there exists a set $X \subset V(G)$ such that $Y=\delta(X)$. A $k$-edge-cut $Y$ is cyclic if all the components of $G-Y$ contain a cycle. Given a plane embedding of a graph $G$, two faces of $G$ are adjacent if they share an edge. A cyclic edge-cut $Y$ is trivial if one of the connected components of $G-Y$ is a cycle.

Zhang and He [20] extended the notion of resonant patterns to all plane graphs. Let $G$ be a plane graph.The unbounded face of $G$ is the outer face, the other faces are the inner faces. Given a perfect matching $M$ of $G$, a face $F$ of $G$ of even size is resonant (with respect to $M$ ) if exactly half of the edges incident with $F$ belong to $M$. A resonant pattern of $G$ is a (possibly empty) set $R$ of pairwise disjoint inner faces such that there exists a perfect matching $M$ of $G$ with respect to which every face in $R$ is resonant. In particular, every face in $R$ is bounded by a cycle of even length. In case $G$ is a fullerene graph, this definition coincides with the one given in the introduction: it suffices to choose a pentagon as the outer face of $G$, since a pentagon is never resonant. Similarly as for fullerene graphs, $\mathscr{R}(G)$ is defined as the set of all resonant patterns of the plane graph $G$.

We end this section by stating Tutte's theorem [12, 18]. A graph (or a connected component of a graph) is odd if it has an odd number of vertices. It is even otherwise.

Theorem 3. A graph $G$ has no perfect matching if and only if there exists a set $S \subset V(G)$ such that the number of odd components of $G-S$ is greater than $|S|$. 


\section{The proof of Theorem 2}

We start with a straightforward observation.

Observation 4. Let $G$ be a cubic bridgeless graph, and let $Y$ be a k-edge-cut of $G$ with $k \in\{2,3\}$. Then $Y$ is cyclic if and only if both $|X|$ and $|V \backslash X|$ are greater than 1 , where $Y=\delta(X)$.

Proof. Set $G^{\prime}:=G[X]$. By the symmetry of the roles played by $X$ and $V \backslash X$, it suffices to prove that $G^{\prime}$ contains a cycle. To see this, note that $G^{\prime}$ has at most one vertex of degree less than 2 , and hence $G^{\prime}$ cannot be a forest.

Since Zhang and He [20] proved that Conjecture 1 is true for fullerene graphs containing adjacent pentagons, we focus on fullerene graphs that satisfy the IPR. In particular, such fullerene graphs have no non-trivial cyclic 5-edge-cut. Indeed, fullerene graphs that contain a non-trivial cyclic 5-edgecut were characterised by Kutnar and Marušič [11, and by Kardoš and Škrekovski [9]: it turns out that those fullerene graphs do contain adjacent pentagons.

We now prove a crucial property of fullerene graphs with no non-trivial cyclic 5-edge-cut.

Proposition 5. Let $G$ be a fullerene graph with no non-trivial cyclic 5-edgecut. Suppose that there is an 8-edge-cut that separates an even subgraph $H \subset G$ from $G-H$, and $G-H$ contains at least three pairwise non-adjacent pentagons. Then $G-H$ has a perfect matching.

Proof. Suppose that $G^{\prime}:=G-H$ does not contain a perfect matching. Then Tutte's theorem ensures the existence of a set $S$ of vertices of $G^{\prime}$ such that $G^{\prime}-S$ contains more than $|S|$ odd components. Let $\mathcal{O}$ be the set of odd components of $G^{\prime}-S$. Since the number of vertices of $G$ is even, $|\mathcal{O}|$ and $|S|$ have the same parity. Thus, since $|\mathcal{O}|>|S|$ we deduce that $|\mathcal{O}| \geq|S|+2$.

The number $e_{G}(S, \mathcal{O})$ of edges between $\mathcal{O}$ and $S$ in $G$ is at most $3|S|$. Moreover, the number of edges between $H$ and $\mathcal{O}$ is at most 8 . Hence,

$$
e_{G}(S \cup H, \mathcal{O}) \leq 3|S|+8 \leq 3|\mathcal{O}|+2 .
$$

Let $O \in \mathcal{O}$. Notice that the number of edges $e_{O}$ between $O$ and $S \cup H$ is odd, for otherwise the subgraph of $G$ induced by $O$ would have an odd number of vertices of odd degrees. Moreover, $e_{O}>1$ because $G$ is bridgeless. Hence, $e_{O} \geq 3$ for each odd component $O \in \mathcal{O}$. In particular,

$$
e_{G}(S \cup H, \mathcal{O}) \geq 3|\mathcal{O}|
$$


So (2) and (3) imply that $|\mathcal{O}|=|S|+2$.

We now prove that $G^{\prime}-S$ has no even components. To see this, suppose on the contrary that $C$ is an even component of $G^{\prime}-S$. By Observation 4 , there are at least 4 edges between $C$ and $S \cup H$. Then, $e_{G}(S \cup H, \mathcal{O}) \leq$ $3|S|+8-4<3|\mathcal{O}| ;$ a contradiction.

Let $s$ be the number of edges of $G[S]$, and $e_{G}(H, S)$ the number of edges with an end-vertex in $H$ and the other in $S$. We observe that $s+e_{G}(H, S) \leq 1$. Indeed, $e_{G}(S \cup H, \mathcal{O}) \leq 3|S|+8-2\left(s+e_{G}(H, S)\right) \leq 3|\mathcal{O}|+2-2\left(s+e_{G}(H, S)\right)$. Hence, $s+e_{G}(H, S) \leq 1$ by $(3)$.

Since $G^{\prime}-S$ has no even components, this last remark implies that $e_{G}(S \cup$ $H, \mathcal{O}) \neq 3|\mathcal{O}|+1=3|S|+7$.

Now observe that each odd component $O \in \mathcal{O}$ such that $e_{O}=3$ is an isolated vertex of $G^{\prime}-S$. Indeed, if $O$ contains a cycle, then $e_{O} \geq 5$ since $G$ is cyclically 5 -edge-connected. Further, if $O$ is a tree then $e_{O}$ is at least twice the number of leaves of $O$. Hence $O$ is a tree with one leaf, i.e. a single vertex.

We consider two cases regarding the value of $e_{G}(S \cup H, \mathcal{O})$.

$e_{G}(S \cup H, \mathcal{O})=3|\mathcal{O}|$. Then $e_{O}=3$ for each $O \in \mathcal{O}$. Consequently, each odd component is an isolated vertex of $G^{\prime}-S$.

Recall that $G[S]$ has at most one edge and $G^{\prime}-S$ has no even components. Thus, $S$ is not a stable set of $G$, for otherwise $G-H$ would be bipartite. Consequently, $G[S]$ contains exactly one edge $e$, and $G-H-e$ is bipartite. But since $G-H$ contains at least three pentagons, $G-H-e$ contains a pentagon; a contradiction.

$e_{G}(S \cup H, \mathcal{O})=3|\mathcal{O}|+2$. Then $S$ is an independent set. Since $e_{O}$ is an odd number greater than 2 for every odd component $O \in \mathcal{O}$, let $O^{\prime}$ be the unique odd component such that $e_{O^{\prime}}=5$. Hence, every odd component other than $O^{\prime}$ is an isolated vertex of $G^{\prime}-S$.

Let us now consider $O^{\prime}$. If $O^{\prime}$ contains a cycle, then since $G$ has no nontrivial cyclic 5-edge-cut, we deduce that $O^{\prime}$ is a pentagon of $G$ (note that $G-O^{\prime}$ cannot be a cycle: $|\mathcal{O}|>1$ since $G-H$ is even, and hence $G-O^{\prime}$ contains vertices of degree 3 ). If $O^{\prime}$ is acyclic, then it is a tree with at most (and hence exactly) two leaves (for otherwise $e_{O^{\prime}}$ would be at least 6 ). Consequently, $e_{O^{\prime}}=5$ implies that $O^{\prime}$ is a path or order 3 .

In both cases $G-H-O^{\prime}$ is bipartite; this is a contradiction since $G-H$ contains at least three pairwise non-adjacent pentagons. 
Let $L$ be the 'lollipop graph' in Figure 1. Proposition 5 immediately implies the following.

Corollary 6. Let $G$ be an IPR fullerene graph, and suppose that $H \subset G$ is a subgraph isomorphic to $L$. Then every perfect matching of $H$ can be extended to a perfect matching of $G$.

Let us point out that Proposition 5 also implies that every perfect matching of a pair of adjacent hexagons of an IPR fullerene can be extended to the whole graph.

The following lemma was proved by Zhang and He [20, Lemma 3.3].

Lemma 7 (Zhang and He). Let $G$ be a connected plane graph with a perfect matching, uv an edge incident with the outer face of $G$ and another face $C$. Then

$\mathscr{R}(G) \subseteq \mathscr{R}(G-u v) \cup \mathscr{R}(G-u-v) \cup\{R \cup\{C\}: R \in \mathscr{R}(G-u v) \cap \mathscr{R}(G-u-v)\}$

Notice that in the last term of the right-hand side of (4), we may require that $R$ be vertex-disjoint from $C$, for otherwise $R \cup\{C\} \notin \mathscr{R}(G)$. Consequently, under the same hypothesis as in Lemma 7, we can write

$$
\begin{aligned}
|\mathscr{R}(G)| \leq & |\mathscr{R}(G-u v) \cup \mathscr{R}(G-u-v)| \\
& +\mid\{R: R \in \mathscr{R}(G-u v) \cap \mathscr{R}(G-u-v) \text { and } V(R) \cap V(C)=\emptyset\} .
\end{aligned}
$$

We shall also use the following theorem of Zhang and He [20, Theorem $2.3]$ in the proof of Theorem 2 .

Theorem 8. For every plane graph $G$,

$$
|\mathscr{R}(G)| \leq \operatorname{pm}(G) .
$$

We formalise the following well-known (and straightforward) fact as an observation.

Observation 9. For every graph $G$ and every edge uv of $G$,

$$
\operatorname{pm}(G)=\operatorname{pm}(G-u v)+\operatorname{pm}(G-u-v) .
$$

Let $I_{h}$ be the graph of the truncated icosahedron, and let $F$ be the graph in Figure 1 consisting of one pentagon and two hexagons. 


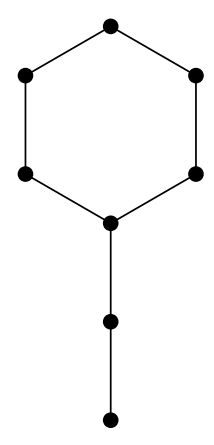

$L$

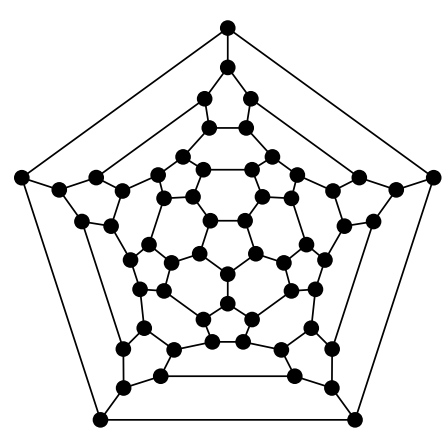

$I_{h}$

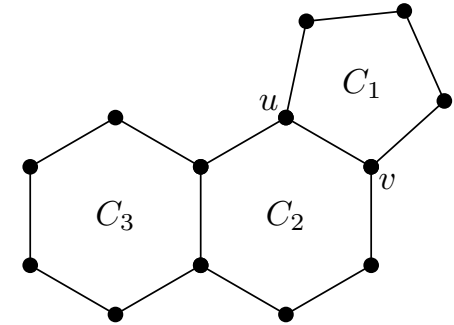

F

Figure 1: The lollipop graph $L$, the truncated icosahedron $I_{h}$, and the graph $F$ in Lemma 10.

Lemma 10. Every IPR fullerene graph other than $I_{h}$ contains a subgraph isomorphic to $F$.

Proof. If $G$ is a fullerene graph that satisfies the IPR and does not contain a subgraph isomorphic to $F$, then every hexagon of $G$ is adjacent to zero or three pentagons. First, let us show that every hexagon of $G$ is adjacent to three pentagons. If not, then there exists a hexagon $H_{0}$ adjacent to zero pentagons and to a hexagon $H_{1}$ that is adjacent to three pentagons (because $G$ is connected and contains pentagons). This would imply that $G$ violates the IPR; a contradiction.

Let $n, e, f$, and $f_{d}$ be the number of vertices, edges, faces and faces of size $d$ of $G$, respectively. Since $G$ is cubic, $3 n=2 e$. Substituting into Euler's formula $(n-e+f=2)$, we obtain $n=2 f-4$. Since every hexagon is adjacent to three pentagons, and there are twelve pentagons altogether, $3 f_{6}=5 f_{5}=60$, so $f=f_{5}+f_{6}=32$, and hence $n=60$. This implies that $G$ is the truncated icosahedron $I_{h}$, and the proof is complete.

We are now ready to prove Theorem 2 .

Proof of Theorem 2. If $G$ violates the IPR, then Zhang and He [20] proved that $B_{G}(1)<\operatorname{pm}(G)$. Now, suppose that $G$ is an IPR fullerene graph not containing $F$ as a subgraph. Lemma 10 implies that $G$ is the truncated icosahedron $I_{h}$. Shiu et al. [17] calculated the sextet polynomial of $I_{h}$, and found that $B_{I_{h}}(1)=5,828$; whereas $\operatorname{Schmalz}$ et al. [15] showed that $\operatorname{pm}\left(I_{h}\right)=$ 12, 500. In particular $B_{I_{h}}(1)<\operatorname{pm}\left(I_{h}\right)$, as required.

So we may suppose that $G$ is an IPR fullerene containing a subgraph isomorphic to the graph $F$ of Figure 1 . The graph $G$ can be embedded in the plane so that the outer face is bounded by the pentagon $C_{1}$. By Corollary 6 , 
there is a perfect matching $M_{1}$ of $G$ such that $C_{3}$ is resonant and $u v \notin M_{1}$, and a perfect matching $M_{2}$ of $G$ such that $C_{3}$ is resonant and $u v \in M_{2}$. In particular, $C_{3}$ is resonant in both $G-u v$ and $G-u-v$. Hence,

$\left|\left\{R \in \mathscr{R}(G-u v) \cap \mathscr{R}(G-u-v): V(R) \cap V\left(C_{3}\right)=\emptyset\right\}\right|<|\mathscr{R}(G-u v) \cap \mathscr{R}(G-u-v)|$.

Therefore, applying (5) to $u v$ and using Theorem 8 and Observation 9 gives

$$
\begin{aligned}
B_{G}(1) & =|\mathscr{R}(G)| \\
& <|\mathscr{R}(G-u v) \cup \mathscr{R}(G-u-v)|+|\mathscr{R}(G-u v) \cap \mathscr{R}(G-u-v)| \\
& =|\mathscr{R}(G-u v)|+|\mathscr{R}(G-u-v)| \\
& \leq \operatorname{pm}(G-u v)+\operatorname{pm}(G-u-v) \\
& =\operatorname{pm}(G),
\end{aligned}
$$

as required. This completes the proof of Theorem 2 .

Acknowledgements. The authors are grateful to Dominik Domin for explaining the basic concepts of valence bond theory.

\section{References}

[1] S. J. Austin, P. W. Fowler, P. Hansen, D. E. Manolopoulos, and M. Zheng. Fullerene isomers of $\mathrm{C}_{60}$. Kekulé counts versus stability. Chem. Phys. Lett., 228(4-5):478-484, 1994.

[2] E. J. Clar. The Aromatic Sextet. John Wiley \& Sons, London, 1972.

[3] P. W. Fowler and D. E. Manolopoulos. An Atlas of Fullerenes. Oxford University Press, Oxford, 1995.

[4] I. Gutman. Some combinatorial consequences of Clar's resonant sextet theory. MATCH Commun. Math. Comput. Chem., 11:127-143, 1981.

[5] I. Gutman and S. J. Cyvin. Introduction to the Theory of Benzenoid Hydrocarbons. Springer-Verlag, Berlin, 1989.

[6] I. Gutman, H. Hosoya, T. Yamaguchi, A. Motoyama, and N. Kuboi. Topological properties of benzenoid systems, III. Recursion relation for the sextet polynomial. Bull. Soc. Chim. Beograd, 42:503-510, 1977.

[7] H. Hosoya and T. Yamaguchi. Sextet polynomial. A new enumeration and proof technique for the resonance theory applied to the aromatic hydrocarbons. Tetrahedron Lett., 16(52):4659-4662, 1975. 
[8] P. E. John, H. Sachs, and M. Zheng. Kekulé patterns and Clar patterns in bipartite plane graphs. J. Chem. Inf. Comput. Sci., 35(6):1019-1021, 1995.

[9] F. Kardoš and R. Škrekovski. Cyclic edge-cuts in fullerene graphs. J. Math. Chem., 44(1):121-132, 2008.

[10] H. W. Kroto, J. R. Heath, S. C. O'Brien, R. F. Curl, and R. E. Smalley. $\mathrm{C}_{60}$ : Buckminsterfullerene. Nature, 318:162-163, 1985.

[11] K. Kutnar and D. Marušič. On cyclic edge-connectivity of fullerenes. Discrete Applied Math., 156(10):1661-1669, 2008.

[12] L. Lovász and M. D. Plummer. Matching theory, volume 121 of NorthHolland Mathematics Studies. North-Holland Publishing Co., Amsterdam, 1986. Annals of Discrete Mathematics, 29.

[13] N. Ohkami, A. Motoyama, T. Yamaguchi, H. Hosoya, and I. Gutman. Graph-theoretical analysis of the Clar's aromatic sextet: Mathematical properties of the set of the Kekulé patterns and the sextet polynomial for polycyclic aromatic hydrocarbons. Tetrahedron, 37(6):1113-1122, 1981.

[14] E. Osawa. Superaromaticity. Kagaku (Kyoto), 25:854-863, 1970.

[15] T. G. Schmalz, W. A. Seitz, D. J. Klein, and G. E. Hite. C 60 carbon cages. Chem. Phys. Lett., 130(3):203-207, 1986.

[16] W. C. Shiu, P. C. B. Lam, F. Zhang, and H. Zhang. Normal components, Kekulé patterns, and Clar patterns in plane bipartite graphs. J. Math. Chem., 31(4):405-420, 2002.

[17] W. C. Shiu, P. C. B. Lam, and H. Zhang. Clar and sextet polynomials of buckminsterfullerene. J. Mol. Struct. (Theochem), 622(3):239-248, 2003.

[18] W. T. Tutte. The factorization of linear graphs. J. London Math. Soc., 22(2):107-111, 1947.

[19] F. Zhang and R. Chen. A theorem concerning polyhex graphs. MATCH Commun. Math. Comput. Chem., 19:179-188, 1986.

[20] H. Zhang and J. He. A comparison between 1-factor count and resonant pattern count in plane non-bipartite graphs. J. Math. Chem., 38(3):315$324,2005$. 